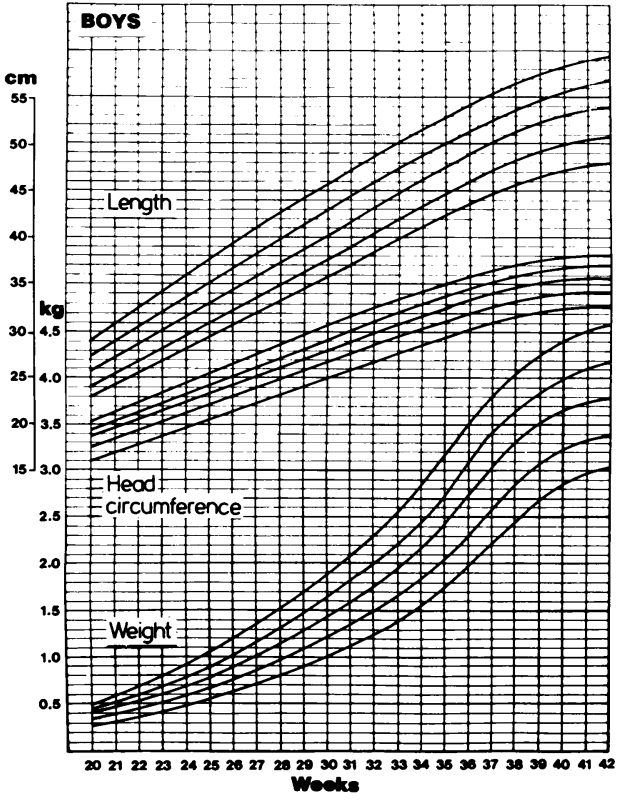

Fig 1 Growth parameters: boys (means $\pm I$ and $2 S D s$ ).

We thank Mrs P Kenyon for her assistance in collecting the data, and Dr RP Petchey for computer processing.

\section{References}

1 Keen DV, Pearse RG. Birthweight between 20 and 42 weeks' gestation. Arch Dis Child 1985;60:440-6.

2 Gairdner D, Pearson J. Revised Gairdner-Pearson growth charts. Arch Dis Child 1985;60:1202.

${ }^{3}$ Kitchen WH, Robinson HP. Dickenson AJ. Revised intra-

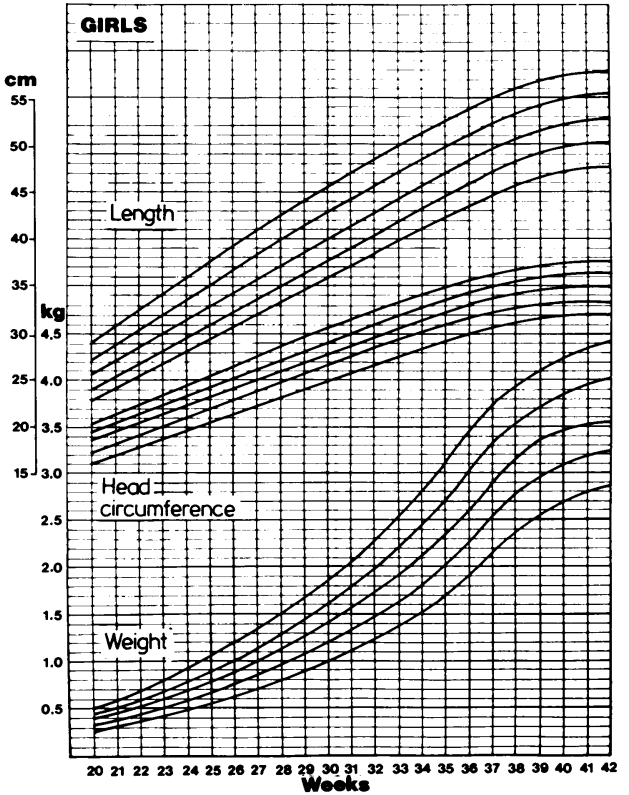

Fig 2 Growth parameters: girls (means \pm 1 and $2 S D s$ ).

uterine growth curves for an Australian hospital population. Aust Paediatr J 1983;19:157-61.

${ }^{4}$ Baum JD, Searls D. Head shape and size of preterm lowbirthweight infants. Dev Med Child Neurol 1971;13:576-81.

5 Gruenwald P. Growth of the human fetus. Am J Obstet Gynecol 1966;94:1112-9.

Correspondence to Dr D V Keen, Department of Paediatrics, Northern General Hospital, Sheffield S5 7AU.

Accepted 3 February 1988

\title{
Pansystolic murmur in the newborn: tricuspid regurgitation versus ventricular septal defect
}

\author{
J R KELLEY AND W G GUNTHEROTH
}

Division of Pediatric Cardiology, Department of Pediatrics, University of Washington School of Medicine, Seattle, USA

SUMMARY Neonates with a pansystolic murmur who had Doppler echocardiography were reviewed. Ten infants had tricuspid regurgitation (detected at a mean age of 25 hours), 12 had a ventricular septal defect (detected at 65 hours), and seven had both. Tricuspid regurgitation is the more likely cause of a pansystolic murmur at the lower left sternal border in the first day of life.
When we began peforming Doppler echocardiography in neonates with murmurs that suggested a ventricular septal defect, we found that many of them had neither an image of a ventricular septal defect nor flow disturbance; instead, we found turbulent systolic flow of tricuspid regurgitation. Further, we had the impression that the infants with tricuspid regurgitation had the onset of murmurs during the first day of life. We decided to investigate this in all newborn infants in our hospital who were 
shown to have a ventricular septal defect or transient tricuspid regurgitation.

\section{Subjects and methods}

All infants born at the University of Washington Hospital over the past four years who had a pansystolic murmur were subjected to the same protocol. This comprised an immediate examination by the admitting nurse, including cardiac auscultation, and by a paediatric resident at once if there was any evidence of distress, but in any case by 12 hours of age. Nursing staff auscultated for heart rate at four hour intervals until the infant was stable, and at eight hour intervals thereafter. Doppler echocardiograms were performed with an Advanced Technology Laboratories Mark 600 echocardiograph. Standard methods were used for detection of the ventricular septal defect ${ }^{1}$ and tricuspid regurgitation. ${ }^{2}$ We did not include in the group with tricuspid regurgitation those infants who had minimal tricuspid regurgitation with a jet found only close to the tricuspid valve and extending less than 1 or $2 \mathrm{~mm}$ into the right atrium, as this degree of tricuspid regurgitation is present in many normal infants and is not audible at the chest wall. The age of each infant in hours at the time of discovery of the murmur was recorded. For infants whose murmur was discovered after 5 days of age, we assigned a maximal figure of 120 hours for purposes of comparison, to avoid excessive weighting by outliers. $t$ Scores were calculated for the means and the confidence level determined using a two tailed table.

\section{Results}

Ten infants had tricuspid regurgitation only; their mean (SD) age at the time of detection of their murmur was $25.2(41.0)$ hours. Twelve infants had a ventricular septal defect only; the mean (SD) age of detection was $65 \cdot 17(31 \cdot 3)$ hours. Seven infants had both a ventricular septal defect and tricuspid regurgitation; the mean (SD) age of detection was $35.93(34.8)$ hours.

The difference between the mean time of detection for the infants with transient tricuspid regurgitation and those with ventricular septal defect was 40 hours $(p<0.02)$. There was a mean 10.7 hour delay for the discovery of the ventricular septal defect + tricuspid regurgitation group after the detection of the tricuspid regurgitation group. The difference in means between the ventricular septal defect+ tricuspid regurgitation group and the ventricular septal defect group, $29 \cdot 2$ hours, almost achieved significance $(p>0 \cdot 05$ but $<0 \cdot 1)$.

\section{Discussion}

Tricuspid regurgitation in the adult or child without pulmonary hypertension produces a murmur that is not high pitched, ${ }^{3}$ and unless there is substantial regurgitation, the murmur will be soft and frequently inaudible, reflecting the normally low pressure gradient between the right ventricle and right atrium. In the presence of pulmonary hypertension the pressure gradient in tricuspid regurgitation may approach or exceed the gradient involved in a ventricular septal defect, resulting in a murmur that is indistinguishable from that of a small ventricular septal defect.

In the newborn, the pulmonary artery pressure is high immediately after birth, falls quickly in the first

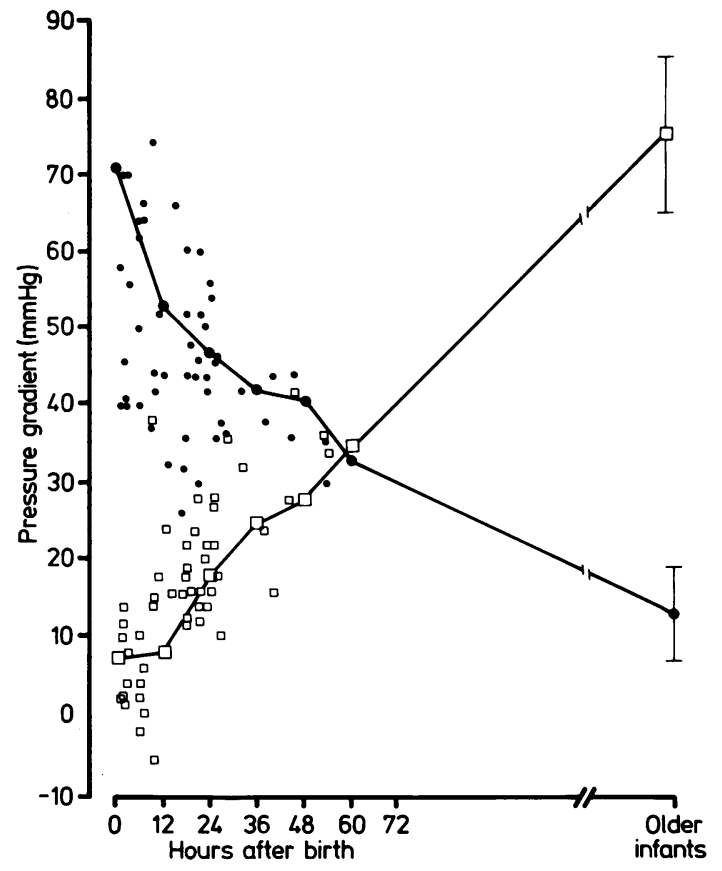

Figure Pressure gradients in normal newborns calculated from data of Emmanouilides et al. ${ }^{4}$ The solid circles represent the systolic gradient between the right ventricle and right atrium in 51 infants studied at the specified age. The open squares represent individual values for the systolic pressure gradient between the left ventricle and right ventricle in the same subjects. The descending line represents the average force that could drive tricuspid regurgitation, and the ascending line the average force that could cause left-to-right flow through a small ventricular septal defect. The large circles and squares on the two lines are the mean values for all points in the preceding 12 hour interval. The last data points, with vertical bars, represent the mean and range of normal for 'newborn' published by Rudolph. ${ }^{5}$ 
3 days, ${ }^{4}$ and continues to fall for several weeks. ${ }^{5}$ The initially high pulmonary artery pressure will increase the pressure gradient between the right ventricle and right atrium. The figure shows a plot of the pressure gradients calculated by us for tricuspid regurgitation in 51 normal infants catheterised during the first 3 days of life. The calculations are based on the 1964 data of Emmanouilides et al ${ }^{4}$ and Rudolph. ${ }^{5}$ During the first 24 hours the mean (SD) normal systolic pressure gradient between the right ventricle and atrium is 53 (12) $\mathrm{mm} \mathrm{Hg}$, and by 60 hours has dropped to 33 (14).

Similarly, plots of pressure gradients between the left ventricle and right ventricle in normal newborn infants calculated by us from the same data ${ }^{4}$ show a very low gradient at birth (figure). During the first 24 hours, the mean (SD) systolic gradient is $8(9) \mathrm{mm} \mathrm{Hg}$, and by 60 hours has risen to $35 \mathrm{~mm} \mathrm{Hg}$. These data provide the physical basis for the delay in detection of the murmur of a ventricular septal defect in the neonate, namely the absence of a significant pressure gradient, even with very small ventricular septal defects.

None of our patients with tricuspid regurgitation had any structural deformity of the tricuspid valve, and none of the murmurs persisted after the neonatal period. Dilatation of the right ventricle and the tricuspid annulus is the most frequent mechanism for tricuspid regurgitation in adults without structural heart disease. ${ }^{6}$ Our undocumented impression was that some degree of stress was common in patients at the time of their transient tricuspid regurgitation.

We conclude that transient tricuspid regurgitation occurs frequently in a hospital with a high risk obstetrical programme, at least as often as ventricular septal defects. It is a more likely cause of a pansystolic murmur in the first day of life than a ventricular septal defect.

\section{References}

' Stevenson JG, Kawabori I, Guntheroth WG. Differentiation of ventricular septal defects from mitral regurgitation by pulsed Doppler echocardiography. Circulation 1977;56:14-8.

${ }^{2}$ Hatle L, Angelsen B. Doppler ultrasound in cardiology. 2nd Ed. Philadelphia: Lea and Febiger, 1985:170-6.

${ }^{3}$ Perloff JK. Physical examination of the heart and circulation. Philadelphia: WB Saunders, 1982:208-14.

${ }^{4}$ Emmanouilides GC, Moss AJ, Duffie ER Jr, Adams FH. Pulmonary arterial pressure changes in human newborn infants from birth to 3 days of age. J Pediatr 1964;65:327-33.

${ }_{5}$ Rudolph AM. Congenital diseases of the heart. Chicago: Year Book Medical Publishers, 1974:29-48.

${ }^{6}$ Mikami T, Kudo T, Sakurai N, SakamotoS, Tanabe Y, Yasuda H. Mechanisms for development of functional tricuspid regurgitation determined by pulsed Doppler and two-dimensional echocardiography. Am J Cardiol 1984;160:160-3.

Correspondence to Dr WG Guntheroth, Department of Pediatrics, RD20, University of Washington, Seattle, Washington 98195, USA.

Accepted 3 May 1988 